\title{
XXXVIII.
}

Aus dem Diakonissenhaus Paulinenstiftung zu Wiesbaden

(Dr. E. Pagenstecher).

\section{Zur Kasuistik und Histologie der kavernösen Muskelangiome.}

\author{
Von \\ Dr. med. Karl Keller, \\ Assistenzarzt.
}

Obwohl die primären kavernösen Muskelangiome zu den relativ seltenen Geschwülsten gehören, hat sich doch die Zahl der Fälle besonders in letzter Zeit bedeutend vermehrt. Ein italienischer Autor namens Alessandri (10)1) konnte 22 Fälle von kavernöser Huskelangiomen mit anderen zahlreichen venösen, arteriellen und capillären sowie Iymyhangiomen zusammenstellen.

Ich bin in der Lage, einen weiteren Fall hinzuzufügen:

M. K., 20 Jahre alt, Dienstmädehen, hat schon von frühester Jugend an eine Gesehwalst an der Hinterseite des rechten Oberschenkels, die ab und zu, meist im Frühjahr und Herbst, Sehmerzen verursachte und dabei etwas anschwoll. Die Größe der Geschwulst habe sich in ihrem Verhältnis zum Oberschenkel nicht geändert.

Status praosens: Sonst gesundes Mädchen in gutem Ernährungszustande.

Auf der Beugeseite des rechten Oberschenkels findet sich eine etwa das mittlere Drittel desselben einnelımende, flach-ovale, äußerst druckempfindliche Geschwulst von anscheinend derb fluctuierender Konsistenz. Sie scheint mit der Yuskelfaszie in inniger Verbindung zu stehen, jedenfalls sitzt sie der Beugemuskulatur des Oberschenkels fest auf und läßt sich gegen dieselbe, auch am Rande nicht vollständig scharf abgrenzen. Die. äuBere Haut über der Geschwulst zeigt normale Beschaffenheit, kam aber nur mit Mühe als Falte emporgehoben werden.

Der Gang ist hinkend, da znr Zeit starke Sclmerzen bestehen. Dabei wird das Knie in mäBiger Bengung etwa in einem Winkel ron $20-30^{\circ}$ gehalten. Vollständige Streckung ist schon wegen der hochgradigen Schmerzhaftigkeit unmöglich.

1) Die mit Klanmern versehenen Ziffern bezichen sich auf das am Schlusse angefügte Literaturverzeichnis. 
Nach 8 tägiger Bettrube ist die Geschwulst nicht mehr schmerzhaft, viel kleiner, die Hant darüber versehieblich.

11. Dezember 1903 Operation in Chloroformnarkose, ohne Blutleere:

Großer Längsschnitt über die Gesehwulst. Haut und subkutanes Fettgewebe erweisen sich von ihr ganz getrennt. Die Aponenrose ist von einer Fettmasse durchsetzt, die sich zwischen $\mathbf{M}$. biceps femoris und M. semimembranosus als ein großer Klumpen hineinerstreckt und medial mit dem $\mathbf{M}$. semimembranosus zusammenhängt. Fettgewebsziige von feinkörnigem, derbem Charakter gehen in den Muskel hinein, denselben fast in seiner ganzen Iänge zwischen den gesunden Fasem durchsetzend nnd nur das zentrale und periphere Ende freilassend.

Ungefähr in der Mitte wird ein karernöses Maschenwerk eröffnet, aus dem venöses, dunkel gefärbtes Blut in mäliger Menge abflieft, das ferner frische und entfärbte, lockere Blutgerinnsel enthält. Zur Entfernung der ganzen Geschwulstmasse muß der Muskel zum größteu Teil entfernt werden. Es bleibt peripher ein quer abgeschnittener Muskelrest, aus dem aber ein Fettzapfen lierausprapariert ist, und zentral das mediane Drittel von dev Aponemose.

In der liefe wird der $\mathrm{N}$. ischiadicus von Fettyewebe umliullt sichtbar, bleibt aber von seiner Hülle bedeckt. Dio Blutung ist gering, hauptsächlich aus den Venen der Muskelsubstanz. Der periphere Muskelstumpf wird an seine zurückgebliebene Faszie angenäht. Sorgfältige Blutstillung. Drain in den oberen Wundwiekel. Sonst Naht und komprimierender Verband.

Bei der Entfernung der Nähte am 19. November 1904 entleerte sich ein großes Hämatom, das späterhin durch cinen Fadenabszeß in Eiterung überging, leichtes Fieber verursachte und so die Hoilung wesentlieh verzögerte. Anfang Januar konnte Patientin mit fast vollständig verheilter Wunde ohne hinkenden Gang nach Hause entlassen werden.

Der dureh die Exstirpation gewonnene Tumor zeigt auf dem Durchschnitt in einer lockeren Fethû́lle ein etwas mehr abgegronztes lipomartiges, aber mit dem Muskel zusammenhängendes Gebilde, dessen pomeranzengroßer Kern aus kavernösem, kleinmaschigem Gewebe besteht, das mit dem oben beschriebenen lipomatösen Gewebe in die Muskelsubstanz direkt übelgeht wd dieselbe allseitig durchsetzt, andererseits sich allmählich gegen das Fettgewebe nach auben hin verliort, so dab es aussieht, als ob das Fett sich in fortschreitender kavernöser Entartung befände.

Zur mikroskopischen Untersuchung wurden an verschiedenen Stellen Stücke herausgeschnitten, in Formalin fixiert und in der gewöhnlichen Weise nach der Paraffinmethode behandelt. Die Schnitte wurden mit Hämatoxylin-Eosin nach van Gieson, und anf elastische Fasern nach Weigert bezw. mit Orcein gefarbt.

Die Huskulatur zeigt folgende Veränderungen: Sie ist durch zwischengewuchertes Fett- und Bindegewebe in einzelne gröbere oder kleinere Bündel abgeteilt. Die einzelnen Bündel sind nun durch den Schnitt teils in der Längs-, teils in der Quer-, teils in der schrägen Richtung getroffen, woraus man schließen mub, daß durch die Fettwucherung eine gegenseitige Verschiebung der Muskelbündel zu einander zustande gekommen ist. Das Fettbezw. Bindegewebe dringt an manehen Stellen auch zwischen die einzelnen 
Nuskelfasern ein und trennt sie ganz oder teilweise voneinander. Die Fasern selbst sind oft verschmälert, dann wieder von normaler Breite, Fasern ganz verschiedenen Kalibers liegen dicht nebeneinander - vielfach geschlängelt oder schraubenförmig gewunden, so daß oft ein und dieselbe Faser in verschiedenen nebeinanderliegenden Schnittrichtangen getroffen ist. Im Lüngsschnitt ist die charakteristische Querstreifung fast ausnahmslos anch bei den dünnsten Fasern dentlich zu erkennen. Ferner aber tritt eine durch fibrilläre Zerklüftung bedingte, intensive, markante Iüngsstreifung hervor, der auf dem Querschnitt eine starke Cohnheim sche Felderung entspricht. Die Sarkolemmkerne zeigen eine mäßige Vermehrung and hie und da Anordnung in Reihen. Die Kerne des Perimysium internum sind ebenfalls vermehrt, oft sehr stark. Was das weitere Schicksal der Muskelfasern anbetrifft, so verschmälern sie sich immer mehr, zerfallen schließlich in kleine längliche oder rundliche, mehrkernige Körperchen, die nur noch schwach, teilweise oder überhaupt nicht mehr gefärbt sind und meist in Gruppen, seltener vereinzelt im Fett- oder Bindegewebe liegen. Es handelt sich also um eine einfache Atrophie.

Der kavernöse Anteil der Geschwulst besteht aus unregelmäßig gestalteten, dureh Pfeiler, Balken und Septen weiter abgeteilten Hohlräumen von ganz verschiedener Größe. Finmal sind sie ganz klein, erweiterten Kapillaren gleichend, dann wieder anffallend groß. Die Septen und Balken ihrerseits sind bald breit, massig oder ganz fein und liegen dann im Querschnitt getroffen oft einzeln als rundliche bindegewebige Gebilde mitten im Hohlraum. Hier und da finden sich Thromben, meist der Wand anliegend, geschichtet und in Organisation begriffen. Yan sieht dentlich, wie rundliche bezw. spindelförmige Zellen mit bläulich gefärbtem Zellleib (Fibroblasten) und dunklerem rundlichen, Nukleolen enthaltendem Kern in den Thrombus hineinwachsen. Der blutig-flüssige Inhalt ist leider bei der Operation ausgeflossen.

Die Wandungen der Hohlräume bieten die mannigfachsten Abwechslungen: Einmal grenzen atrophische, auch noch gat erbaltene Muskelbündel oder Fasern direkt ohne Endothel nackt an die Hohlräume, dann finden sich wieder solche mit Endothel umsäumt oder auch von einem schmäleren. oder breiteren byalinen Bindegewebsstreifen, der nun seinerseits wicder mit Endotbel besetzt sein kann oder auch nicht. Ähnlich verhält sich das angrenzende Fett- und Bindegewebe, welch letzteres auch die Septen, Pfeilerund Balken bildet. Je weiter man dem Zentrum der Geschwulst zu kommt, desto mehr bestehen die Wandungen aus dickem hyalinen Bindegewebe, welches in den van Gieson-Präparaten intensiv rot gefärbt ist, in Lücken aber gelb gefärbte Züge enthält.

Die elastischen Fasern finden sich als feine schraubenförmig gewundene Fädchen durch die ganze Geschwulst verbreitet, meist im Bindegewobe sitzend, dann aber auch, dem Durchschnitt von Platten entsprechend, als dicke dunkle Stränge, teils parallel den Lumina der Hohlränme, teils aber: auch ohne jede Beziehung durch das ganze Präparat laufend.

An wenigen Stellen findet sich glatte Muskulatur teils in wirbelartiger Anordnung; an anderen Stellen erinnert der Bau der Wandungen an den kleiner Venen: $\mathrm{Zu}$ innerst findet sich das Endothel mit einer dünnen Schicht elastischer Fasern, die Media fehlt; der Adventitia entsprechend sieht man. 
dann quer getroffene, in Bündeln liegende glatte Muskelfasern (nach van Gieson gelb gefärbt) durch Bindegewebe voneinander in ziemlich regelmäßigen Abständen getrennt.

Wir haben also somit:

1. wandungslose Hohlräume ohne Endothel, direkt an das nackte Muskel-, Fett- oder Bindegewebe grenzend, dann

2. solche mit Endothel aber ohne weitere Wand: kapilläre Holılräume,

3. Bindegewebige Wandungen mit Endothel: kavernöse Hohlränme,

4. solche mit dem Bau nach Venen gleichenden Wandungen: Venöse Hohlräume.

Die kavernösen überwiegen ganz beträchtlich.

Demnach liegt also ein kavernöses Muskel-Angiom vor, wie es von zahlreichen Autoren (E. Gurlt(1), zuletzt von P'u porac (4), Honsell (7) und Riethus (9) in ähnlicher Weise beschrieben worden ist.

Es ist in vielen Fällen angeboren, in anderen kurz nach der Geburt oder wenigstens im Laufe des 1. Lebensjahrzehntes entdeckt worden. Fur im Falle Honsells (8) und Demarquays (1) wurde der Tamor relativ spät im 19. bezw. 15. Tebensjahr entdeckt. Einmal soll sich die Geschwulst in Anschlub an ein Trauma: Fall auf den linken Ellenbogen entwickelt haben (Bayta (6). Im allgemeinen wächst sie langsan und bleibt meist latent, bis sie aus irgend welchen Crsachen, die wir nicht näher kennen, anfängt, rascher zu wachsen Morgan (1, 5, 6, 9) bezw. nicht unerhebliche Beschwerden zu machen. In unserem Falle bestand der Tumor schon seit Jugend und machte wie auch anscheinend im Falle $\mathrm{H}$ onsells ( 7 ) periodisch auftretende Beschwerden, die wohl auf entzündliche Vorgänge verbunden mit Bildung und Organisation von Thromben im Tumor auf oder äußere Einwirkungen, Insulte u. dgl. zurückzuführen sind.

Was den Sitz der Geschwulst anbelangt, so ist eine mäßige Bevorzugung der unteren Extremität bezw. des Oberschenkels nicht zu verkennen. Sie sitzt sclten circumseript (d e M organ (1), Liston (1), Ri ethus Fall 2 (9) häufiger diffus im Muskel, tritt auch hier und da in Beziebung mit dem umgebenden Fettgewebe und Bindegewebe, so daß es oft schwer ist, den primären Sitz zu entscheiden. In unserem Falle war die Geschwalst von ciner lockeren Fetthülle umgeben, sie selbst stellte äußerlich ein lipomartiges mit dem Muskel zusammenhängendes Gebilde dar, dessen Kern aus kavernösem Gewebe bestand. Da nun aber das Fettgewebe keinerlei Läppchen- 
bildung erkennen läßt, so ist wobl ein echtes Lipom auszuschließen und ein primärer Sitz im Muskel anzunehmen. Diese Auffassung: wird bestätigt durch folgende Ausführungen M uskatellos (2): „Sehr häufig ist es nicht leicht in jenen Fällen, in welehen das subkutane Gewebe reichlich vorhanden ist, mit Genauigkeit zu entseheiden, ob die Geschwulst im Muskel oder in andern tiefen Geweben ihren Sitz hat. Gelingt es aber festzustellen, daß der Muskel der Sitz der Geschwulst ist, so gewinnt man einen sicheren Orientierungspunkt für die Liagnose. Das Li pom muß für die Mehrzahl der Fälle ansgeschlossen werden, weil es außerordentlich selten vorkommt, so zwar, daß Parmentier gesagt hatte, daf, so oft eine primäre Geschwulst im Muskel gefunden wird, das Lipom auszuschließen ist." Auch im I. Falle Pupovac' (4) geht das subkutane Gewebe in die vom Fettgewebe durchwachsene Yuskulatur ohne scharfe Grenze über. Yan muß wobl annchmen, daß die Geschwulst im Muskel sich der Dicke nach ausdehnend, nach der Tiefe größeren Widerstand findet, während sie der freien Körperoberfläche zustrebend hier wenig behindert ist, dabei aber das umliegende Gewerbe, intramuskuläres, wie sublkutanes, mit zur Wacherung bringt, so daß es ein Lipom vortäuscht.

Die Operation, in der Regel die Exstirpation, bot hier keine größeren Schwierigkeiten. Wenn die Geschwulst sehr ausgedehnt ist und mehrere Maskeln and Muskelgruppen ergriffen hat, muß eventuell die Amputation der befallenen Extremität ausgeführt werden; so z. B. im Falle Riethus (9), wo nach vergeblichem konservativem Vorgehen - die Geschwulst hatte in 2 Jahren die $\mathrm{Mm}$. flexor hallucis. longus, tibialis postic, plantaris und flexor digitor. longus ergriffen - zur Beseitigung der beträehtlichen Beschwerden die Unterschenkelamputation gemacht werden mußte. Die Heilung erfolgt nicht immer glatt wie z. B. in Baytas Fällen (6), wo kavernöses Gewebe zurückgelassen werden mußte, weshalb er annimmt, daß im kavernösen Gewebe entstandene Thromben wenigstens teilweise in den Blutkreislauf gelangen, und so das Fieber und die Störung des Allgemeinbefindens hervorgerufen haben. In unserem Falle war durch Nachblutung ein Hämatom entstanden, das seinerseits wieder dnrch Stichkanalinfection vereiterte.

Die Struktur des eigenen Falles zeigt im allgemeinen eine wesentliche Übereinstimmung mit den diesbezüglich genauer beschriebenen $\mathrm{P}$ a pova c (4) H o n sell (7) R i eth us (9). Die Veränderungen der Yuskulatur, die Begrenzungen der Hohlräume sind sich auffallend ähnlich. Die quergetroffenen von Bindegewebe umwachsenen glatten Muskelfasern, die ich mit der Wucherung der Adventitia 
kleiner Venen in Zusammenhang bringe, vergleicht $\Pi$ onsell $(\tau)$ in seinem Präparat mit den allseitig von Bindegewebe umgrenzten Epithelzapfen eines Carcinoms. Riethus (9) fand an Stelle von Arterien zugehörenden Venen unregelmäßig gestaltete Räume mit muskulöser Wandung and durch partielle Wucherung der Muscularis und Adventitia entstandene leistenartige Vorsprünge in die Lichtung, Gebilde, die bisher noch nicht beschrieben worden sind.

Rundzellenanhäufungen in der Umgebung der Gefäße und Pblebolithen, wic sie Puporac (4) und Honsell (7) gesehen, konnte ich in meinen Präparaten nieht entdecken; ebensowenig wie Wucherungsvorgänge an den Septen oder Zellanhäufungen an Stellen mit reichlichen Septen, wie Riethus (9) beschreibt. - Honsell (7) findet wie auch ich nur zum Teil Endothelbelag der Wandangen.

Nach Ribbert (8) ist das Angiom ein selbständiger aus sich heraus wachsender Gefäßbezirk. Das Wachstum ist aber sekundär. Beim Muskelangiom muß man also eine Entstehung aus dem Gefäßnetz des Muskels innerhalb eines ganz bestimmten Bezirkes annehmen. Das Wachstum bleibt nun selten circumscript, wird vielmehr in den meisten Fällen ein diffuses (s. Literatur). Bei den diffusen werden die ursprünglichen Grenzen durchbrochen und die Muskulatur schrankenlos befallen. Ob ein längere Zeit circumscriptes nachträglich in ein diffuses übergehen kann, bleibt dahingestellt. Ein Ausgangspunkt war nicht immer zu sehen. Unser Fall spricht sehr dafür, da bei ihm der kavernöse Anteil etwa die Mitte des Muskels einnimmt, die beiden Enden freibleiben und der centrale Teil der Geschwulst einen älteren Charakter: Vorwiegen der bindegewebigen Wandungen und Septen trägt. Circumscripte lassen sich leicht ausschälen.

Ein circumscriptes Maskelangiom kann wohl auch vorgetäuscht werden: so z. B. ließ sich bei einem früher operierten ebenfalls 20 jährigen Mädchen die walnußgroße Geschwulst, die rechts dicht neben dem IV. und $\mathrm{V}$. Brustwirbeldornfortsatz in der Tiefe der Muskeln saß, leicht von der Umgebnng stumpf ausschälen und riß nur an kleinen Stellen ein. Durch den Zwischenraum zweier Wirbel ging eine venöse Verbindung, welche einem Stiel zu entsprechen schien. Es zeigte sich aber, daß ein ganzer Strang des Musculus multifidus ausgeschält war, der das Cavernom barg. Die Geschwulst bot im $\ddot{\text { Cb} b r i g e n ~ d i e s e l b e n ~ V e r a ̈ n d e r u n g e n ~ d e r ~ M u s k u l a t u r, ~ w e n n ~ a u c h ~}$ nicht so hochgradig wie im I. Falle. Die Hohlränme waren ebenfalls unregelmäßig gestaltet, zeigten aber Wandungen von vorwiegend varicös venösem Charakter mit glatten Muskelfahren und elastischen Platten und enthielten auch 2 Phlebolithen. 
580 XXXVIII. KELLER, Zur Kasuistik u. Histologio d. kavern. Muskelangiome.

Nach Billroth (cit. bei Gurlt (1) und Petersen (3) kommen die Gefäßgeschwülste unter 3 Formen in den Muskeln vor: I. als Geschwülste von dem Aussehen eines varicösen Zustandes der Venen, ohne Verbindung mit dem Muskelgewebe, in welchem sie liegen, jedoch nicht durch eine bestimmte Bedeckung von Bindegewebe isoliert. II. Ercktiles Gewebe gebildet im Muskelgewebe auf dasselbe übergehend und nicht von demselben bestimmt abgegrenzt. III. Erektiles, einen bestimmten Tumor bildendes Gewebe mit einer Cmbüllung von Bindegewebe, das nicht mit der Muskelsubstanz, in welcher es liegt, zusammenhängt. Auch nach seiner Ansicht haben diese Formen sehr wahrscheinlich einen gemeinsamen Ursprung. Unser I. Fall würde zu der unter II. genannten Form gehören, der zuletzt erwähnte vielleicht zu L., obwohl die Muskulatur schon Veränderungen der Atrophie zeigt. Muskatel lo unterscheidet capilläre, arterielle, venöse und kavernöse Formen.

Was nun die starke Fettwucherung in unserem Falle betrifft, so muß man unterscheiden zwischen den die Muskulatur durchsetzenden Fettgewebe, das in sämtlichen anderen Fällen auch beschrieben ist, und dem das Kavernom umbüllenden, die Fascie durebsetzenden Fettgewebe, welches in unserem Falle eine auffallend selbständige Entwicklung genommen hat. Ersteres würde sich eventuell so erklären lassen, daß Fett an Stelle von atrophischem Muskel tritt. Letzteres jedoch bedarf der Annahme einer primären, der Angiombildung voraus- oder mindestens gleichzeitig gehenden Wucherung. $\mathrm{Da}$ nun anch der intramuskuläre Teil kontinuierlich mit dem übrigen zusammenhängt, dürfte wohl überhaupt dem Fettgewebsanteil der Geschwulst eine gemeinsame, selbständige Bedeutung zukommen, letztere somit als Fibrolipoangiom gedeutet werden.

\section{Literaturverzeichnis.}

1) E. Gurlt, Jahresbericht für 1863-65. Archiv f. klin. Chir, 8. Bd. S. $264 \mathrm{ff}$.

2) Ml uskat ello, Arehiv f. path. Anatomie. 1694. 135. S. 277. Nr. 10.

3) Petersen, Inaug.-Dissert. Kiel 1894.

4) Pupovac, Archiv f. klin. Chirurgio. 1897. 54. Bd. S. 555. Nr. 22.

5) Steele, Brit. med. Jolunal. 432. 1898.

6) Bayta, Deutsche Zeitschr. f. Chirurgie. 1899. s. 200 . Nr. 51.

7) Hon sell, Beiträge z. klin. Chirurgie. 1902. 32. S. 259.

s) Ribbert, Geschwulstlehre. 1903. Angiome.

9) Riethus, Beiträge z. klin. Chirurgie. 1904. S. 454. Nr. 42. Ref. in Zentralbl. f. Chirurgie.

10) Alessandri, Ln caso di angioma cavernoso del musculo trapezio (Poliklinico Sez. chir. 1904. No. 3). 